\title{
Causal Structure and Gravitational Waves in Brane World Cosmology
}

\author{
Kiyotomo Ichiki ${ }^{1,2 *}$ and Kouji Nakamura ${ }^{3,4 \dagger}$ \\ ${ }^{1}$ Department of Astronomy, University of Tokyo, \\ 7-3-1 Hongo, Bunkyo-ku, Tokyo 113-0033, Japan \\ ${ }^{2}$ Division of Theoretical Astrophysics, National Astronomical Observatory, 2-21-1, Osawa, Mitaka, Tokyo 181-8588, Japan \\ ${ }^{3}$ Department of Astronomical Science, the Graduate University for Advanced Studies, \\ 2-21-1, Osawa, Mitaka, Tokyo 181-8588, Japan and \\ ${ }^{4}$ Department of Physics, Hiyoshi Campus, Keio University, Hiyoshi Yokohama, 223-8521, Japan
}

(Dated: October 31, 2018)

\begin{abstract}
The causal structure of the flat brane universe of RSII type is re-investigated to clarify the boundary conditions for stochastic gravitational waves. In terms of the Gaussian normal coordinate of the brane, a singularity of the equation for gravitational waves appears in the bulk. We show that this singularity corresponds to the "seam singularity" which is a singular subspace on the brane universe. Based upon the causal structure, we discuss the boundary conditions for gravitational waves in the bulk. Introducing a null coordinate, we propose a numerical procedure to solve gravitational waves with appropriate boundary conditions and show some examples of our numerical results. This procedure could be also applied in scalar type perturbations. The problem in the choice of the initial condition for gravitational waves is briefly discussed.
\end{abstract}

PACS numbers: $04.50 .+\mathrm{h}, 98.80 . \mathrm{Cq}, 98.80 .-\mathrm{k}$

\section{INTRODUCTION}

Since the proposal of a brane world model of our spacetime by Randall and Sundrum [1] (RS), the phenomenology of brane world cosmological models has been the subject of intensive investigations in recent years. In these brane world models, our universe is regarded as a four dimensional boundary (brane) in a higher dimensional spacetime (bulk). Many authors have found more realistic models which include matter fields on the brane and realize the cosmic expansion [2], and tried to constrain on these models by the observational data.

Due to the recent developments in the technology of astronomy, cosmological density perturbations and their observations through large scale structure and cosmic microwave anisotropies (CMB) have become the most stringent test to constrain on models beyond standard cosmologies. In order to examine the constraints on the brane cosmologies by the CMB observations, we have to know the 5-D informations about perturbations on the entire spacetime including the bulk [3, 4].

In addition to the constraint by the CMB, the stochastic gravitational waves will be a promising candidate which provides a direct and even deeper test of such cosmologies. One might see in principle earlier universe than the photon last scattering epochs by gravitational waves. Although equations for gravitational waves are simpler than those of density perturbations, the problem is essentially the same, i.e, one needs to clarify the evolution of gravitational waves not only on the brane but also in the bulk. Therefore, it is also instructive to clarify the

\footnotetext{
*E-mail address: ichiki@th.nao.ac.jp

${ }^{\dagger}$ E-mail address: kouchan@th.nao.ac.jp
}

minimum information to obtain the evolution of gravitational waves before trying to clarify that of density perturbations in brane cosmologies.

In order to give theoretical predictions of stochastic gravitational waves, many authors have adopted the Gaussian normal (GN) coordinate system in the neighborhood of the brane. In this GN coordinate system, the metric is given by the form

$$
d s^{2}=-\frac{\psi^{2}(\tau, w)}{\varphi(\tau, w)} d \tau^{2}+\varphi(\tau, w) a^{2}(\tau) d \Sigma_{K}^{2}+d w^{2},
$$

and the equation of gravitational waves has the same form as that of the five-dimensional massless scalar field:

$$
\square_{5} h=0 .
$$

The explicit forms of functions in the metric are given in the main text (see Sec. III). To obtain the theoretical spectrum of stochastic gravitational waves, we just solve this equation with appropriate boundary conditions. However, Eq. (2) in terms of the coordinate system (11) includes a singularity at $w=w_{h}$ in the bulk, where the metric function $\varphi$ vanishes. The treatment of Eq. (2) near the singularity is one of difficulties when we obtain the evolution of stochastic gravitational waves [5]. In fact, this singularity corresponds to the "seam singularity" discussed by Ishihara [7].

The aim of this paper is to propose a numerical procedure to solve the evolution of cosmological gravitational waves avoiding the above difficulty in GN coordinate system. The procedure proposed here is based on the characteristic initial value problem according to the causal structure of the entire spacetime. This idea is analogous to the analysis of gravitational waves from a nonspherical domain wall by the one of the authors [8]. We use a null coordinate instead of the proper time on the 
brane. In this procedure, the boundary conditions in the bulk are replaced by the initial condition on a null hypersurface and the above difficulty in the treatment of Eq. (2) near the singularity $w=w_{h}$ is resolved if we simply specify the initial spectrum on a null hypersurface.

The organization of this paper is as follows: In Sec. II we briefly review the global structure of a brane world universe and clarify the region covered by the GN coordinate in terms of the closed chart of the five-dimensional anti-de Sitter spacetime $\left(\mathrm{AdS}_{5}\right)$. In Sec. III we discuss the null hypersurface to clarify the causality of the propagation of gravitational waves in the bulk. In Sec. IV] we develop the formulation to obtain the numerical solutions to Eq. (2) and show numerical examples of solutions which are derived by this formulation. The final section (Sec. V) is devoted to summary and discussions.

Throughout this paper, we consider the model without "dark radiation" following discussions in Ref. [9] and we only consider the flat Friedmann-Robertson-Walker (FRW) brane universe which is supported by recent precise measurements of the CMB 10].

\section{GN COORDINATE SYSTEM IN AdS BULK $_{5}$}

In this section, we first relate GN coordinate system to the flat chart in $\mathrm{AdS}_{5}$, and then consider the correspondence of GN coordinate system and the closed chart in $\mathrm{AdS}_{5}$ which covers the entire $\mathrm{AdS}_{5}$. Through clarifying these relations, we can easily see that the region which is covered by GN coordinate system in the entire $\mathrm{AdS}_{5}$. Secondly, we find where the singularity of the equation (2) is in the bulk.

Now, we consider a brane universe embedded in the $\mathrm{AdS}_{5}$ with a negative cosmological constant $\Lambda_{5}=-4 / l^{2}$. In terms of the static charts of $\mathrm{AdS}_{5}$, the metric on $\mathrm{AdS}_{5}$ is given by

$$
d s^{2}=-f_{K}\left(r_{K}\right) d t_{K}^{2}+f_{K}\left(r_{K}\right)^{-1} d r_{K}^{2}+r_{K}^{2} d \Sigma_{K}^{2},
$$

where $K$ takes the values $-1,0$, and +1 , corresponding to the negative, zero, and positive constant curvature of a maximally symmetric three dimensional space, respectively (for example, see Ref. [7]). The function $f_{K}\left(r_{K}\right)$ in this metric is defined by

$$
f_{K}\left(r_{K}\right):=K+\frac{r_{K}^{2}}{l^{2}}
$$

When $K=+1$ and 0 , the metric $d \Sigma_{K}^{2}$ is given by

$$
d \Sigma_{K}^{2}= \begin{cases}d \chi_{+}^{2}+\sin ^{2} \chi_{+}\left\{d \theta^{2}+\sin ^{2} \theta d \phi^{2}\right\} & (K=+1) \\ d \chi_{0}^{2}+\chi_{0}^{2}\left\{d \theta^{2}+\sin ^{2} \theta d \phi^{2}\right\} & (K=0),\end{cases}
$$

respectively. Though we may consider the case $K=-1$ which corresponds to the open FRW model, we do not treat this case in this paper. In these static charts, a trajectory of three brane is given by $r_{K}=r_{K}\left(t_{K}\right):=$ $a(\tau)$ and $t_{K}=t_{K}(\tau)$, where $\tau$ is the proper time of the world volume of the brane and $a(\tau)$ is a cosmological scale factor on the brane. The equation of the brane motion is given by the generalized Friedmann equation [2]

$$
H^{2}=\left(\frac{\dot{a}}{a}\right)^{2}=\frac{8 \pi G_{\mathrm{N}}}{3} \rho-\frac{K}{a^{2}}+\frac{\Lambda_{4}}{3}+\frac{\kappa_{5}^{4}}{36} \rho^{2},
$$

where the dot denotes the derivative with respect to $\tau$, $\rho$ is the energy density on the brane, $\Lambda_{4}=\kappa_{5}^{4} \lambda^{2} / 12+$ $3 \Lambda_{5} / 4$ is the cosmological constant induced on the brane, $G_{\mathrm{N}}=\kappa_{5}^{4} \lambda / 48 \pi$ and $\kappa_{5}$ are the four-dimensional and fivedimensional gravitational constants, respectively. Here and hereafter we assume $Z_{2}$ symmetry across the brane.

Cosmological solutions of the RS type brane world in terms of GN coordinate system were found by several authors [2], which is given by Eq. (11). In this metric, functions $\psi$ and $\varphi$ are given by

$$
\varphi(\tau, w)=\frac{1}{4}\left[(1-A) e^{\frac{w}{l}}+(A+1) e^{-\frac{w}{l}}\right]^{2}
$$

and

$$
\psi(\tau, w)=\varphi(\tau, w)+\frac{1}{2 H}\left(\frac{\partial \varphi}{\partial \tau}\right)_{w, x^{i}},
$$

where $A:=\sqrt{1+l^{2} H^{2}}$. We have chosen the coordinate $w$ so that $w=0$ on the brane. As noted in Sec. I this coordinate system has a coordinate singularity at $w=$ $w_{h}$, where $w_{h}$ is determined by the equation $\varphi\left(\tau, w_{h}\right)=0$ for each $\tau$. This equation yields

$$
\exp \left(2 \frac{w_{h}}{l}\right)=\frac{A+1}{A-1}
$$

This is nothing but the singularity in Eq. (2).

To give the explicit coordinate transformations between the metrics (11) and (3) with $K=0$, we show the explicit forms of the functions $\left(t_{0}, r_{0}\right)$ in terms of $(\tau, w)$. The function $r_{0}$ in Eq. (3) corresponds to the volume element on the $\tau=$ const. hypersurface in the brane. Then, as shown in Ref. 11], this function is given by

$$
r_{0}^{2}=\varphi(\tau, w) a^{2}(\tau) .
$$

The cosmological scale factor $a(\tau)$ is given by $a(\tau):=$ $r_{0}(\tau, w=0)$. On the other hand, the explicit expression of $t_{0}$ as a function of $(\tau, w)$ is given by

$$
t_{0}-t_{b}=\frac{l^{2}}{a} \sqrt{\frac{A+1}{A-1}}\left[1-\frac{2}{(1-A) e^{2 \frac{w}{l}}+1+A}\right],
$$

where $t_{b}(\tau)$ is chosen so that $t_{0}=t_{b}(\tau)$ on the brane $(w=0)$ for any $\tau$. The derivation of this expression (10) is shown in Appendix A Clearly, the singularity in the equation (2) is just on the region $r_{0}=0$ in the flat chart. Actually, in the vicinity of the singularity $\left(w \sim w_{h}\right), r_{0}$ and $t_{0}$ behave as

$$
\begin{aligned}
& r_{0}=\frac{a(\tau)}{2} \sqrt{\frac{A-1}{A+1}}\left(\xi+\frac{1}{2(A+1)} \xi^{2}\right)+O\left(\xi^{3}\right), \\
& t_{0}=t_{b}+\frac{l^{2}}{a(\tau)} \sqrt{\frac{A+1}{A-1}}\left(-\frac{2}{\xi}+1\right)
\end{aligned}
$$


where $\xi:=(1-A) e^{2 \frac{w}{l}}+1+A$. To find where is the singularity in Eq. (2) in the bulk, we first see the region of $r_{0}=0$ in the entire $\mathrm{AdS}_{5}$ using the closed chart. On this closed chart, we can easily specify the point of the singularity in Eq. (2) in $\mathrm{AdS}_{5}$ bulk by tracing the spacelike geodesic which normal to the brane for each $\tau$.

Now, we consider the relation between the flat chart and the closed chart of $\mathrm{AdS}_{5}$. Though this is already given by Ishihara [7], we repeat his arguments to find the event $\varphi\left(\tau, w_{h}\right)=0$ for each $\tau$ in the closed chart. The $\mathrm{AdS}_{5}$ is identified with the universal covering space of a hyperboloid,

$$
-Y_{0}^{2}-Y_{1}^{2}+\sum_{i=2}^{5} Y_{i}^{2}=-l^{2},
$$

in six-dimensional flat spacetime with the metric,

$$
d s^{2}=-d Y_{0}^{2}-d Y_{1}^{2}+\sum_{i=2}^{5} d Y_{i}^{2}
$$

The flat chart and the closed chart (Eq. (3)) cover this hyperboloid as follows: For the $K=+1$ case (the closed chart),

$$
\left(\begin{array}{c}
Y_{0} \\
Y_{1} \\
Y_{2} \\
Y_{3} \\
Y_{4} \\
Y_{5}
\end{array}\right)=\left(\begin{array}{c}
\sqrt{l^{2}+r_{+}^{2}} \sin \left(t_{+} / l\right) \\
\sqrt{l^{2}+r_{+}^{2}} \cos \left(t_{+} / l\right) \\
r_{+} \cos \chi_{+} \\
r_{+} \sin \chi_{+} \cos \theta \\
r_{+} \sin \chi_{+} \sin \theta \cos \phi \\
r_{+} \sin \chi_{+} \sin \theta \sin \phi
\end{array}\right)
$$

For the $K=0$ case (the flat chart),

$$
\left(\begin{array}{c}
Y_{2}+Y_{0} \\
Y_{2}-Y_{0} \\
Y_{1} \\
Y_{3} \\
Y_{4} \\
Y_{5}
\end{array}\right)=\left(\begin{array}{c}
r_{0} \\
\left(\frac{t_{0}^{2}}{l^{2}}-\chi_{0}^{2}\right) r_{0}-\frac{l^{2}}{r_{0}} \\
-\frac{t_{0}}{l} r_{0} \\
r_{0} \chi_{0} \cos \theta \\
r_{0} \chi_{0} \sin \theta \cos \phi \\
r_{0} \chi_{0} \sin \theta \sin \phi
\end{array}\right) .
$$

We should note that the flat chart does not cover the entire $\mathrm{AdS}_{5}$ since $Y_{2}+Y_{0} \geq 0\left(r_{0} \geq 0\right)$. Comparing Eqs. (15) with Eqs. (16), we found the coordinate transformations from the flat chart to the closed ones:

$$
\begin{aligned}
& r_{+}^{2}=\frac{1}{4}\left(r_{0}-\frac{l^{2}}{r_{0}}+\left(\frac{t_{0}^{2}}{l^{2}}-\chi_{0}^{2}\right) r_{0}\right)^{2}+r_{0}^{2} \chi_{0}^{2}, \\
& \cos \chi_{+}=\frac{r_{0}}{2 r_{+}}\left[1+\frac{t_{0}^{2}}{l^{2}}-\chi_{0}^{2}-\frac{l^{2}}{r_{0}^{2}}\right], \\
& \sin \chi_{+}=\frac{r_{0} \chi_{0}}{r_{+}}, \\
& \tan \left(\frac{t_{+}}{l}-\pi \Theta\left(t_{0}\right)\right)=-\frac{l}{2 t_{0}}\left(1+\frac{l^{2}}{r_{0}^{2}}-\frac{t_{0}^{2}}{l^{2}}+\chi_{0}^{2}\right),
\end{aligned}
$$

where $\Theta\left(t_{0}\right)$ is the step function, i.e., $\Theta\left(t_{0}\right)=0$ for $t_{0} \leq 0$ and $\Theta\left(t_{0}\right)=1$ for $t_{0}>0$.

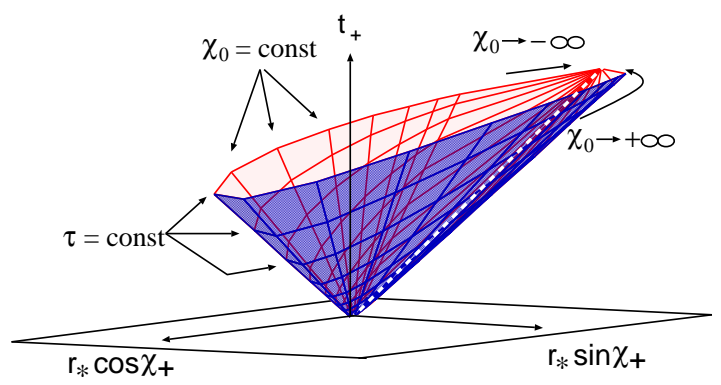

FIG. 1: Embedded flat FRW brane. Hypersurfaces at which $\tau$ or $\chi_{0}$ are constant are also shown in the figure.

In addition to these coordinate systems, it is convenient to introduce the temporal-radial subspace which makes the metric conformally flat

$$
\begin{aligned}
d s^{2} & =f_{+}\left(r_{+}\right)\left(-d t_{+}^{2}+d r_{*}^{2}\right)+r_{+}^{2} d \Sigma_{+}^{2}, \\
\left(r_{*} / l\right) & =\arctan \left(r_{+} / l\right)
\end{aligned}
$$

for the purpose of the investigation of the causal structure. Note that in these coordinates, radial null rays are represented by straight lines at \pm 45 degree. In the coordinate system $\left(t_{+}, r_{*} \cos \chi_{+}, r_{*} \sin \chi_{+}\right)$, the flat FRW brane is embedded as shown in Fig. 10 The $\tau=$ const and $\chi_{0}=$ const hypersurfaces on the flat FRW brane is also shown in the same figure, respectively.

Through the relations (9), (10), and (17), we can see how $\tau=$ const. hypersurfaces in the coordinate system (11) foliate the entire $\mathrm{AdS}_{5}$ bulk. To do this, we have to consider the spacelike geodesics normal to the brane specifying their starting point on the brane. Note that on each $\tau=$ const. hypersurface in the brane $(w=0)$, both functions $t_{0}(\tau)$ and $r_{0}(\tau)$ are also constant, while the other coordinates on the brane are arbitrary. Therefore, we may choose a point $\left(t_{0}, r_{0}\right)$ on the flat chart as a starting point of these spacelike geodesics normal to the brane. Fixing the proper time $\tau$ on the brane, the coordinate functions $r_{+}, t_{+}$, and $\chi_{+}$behave as

$$
\begin{aligned}
r_{+} & =\frac{l^{2}}{a(\tau)} \frac{A}{\sqrt{A^{2}-1}}+t_{b}, \\
\frac{t_{+}}{l} & =\arctan \left[\frac{r_{+}}{l}\right]=\frac{r_{*}}{l}, \\
\cos \chi_{+} & =-1
\end{aligned}
$$

in the limit $w \rightarrow w_{h}(\varphi(\tau, w) \rightarrow 0)$. This shows that the coordinate singularity at which $\varphi\left(\tau, w_{h}\right)=0$ is just on the "seam singularity" $t_{+}=r_{*}, \cos \chi_{+}=-1$ (see Fig. 22).

Note that the seam singularity can be replaced by the other regular portion of a spacetime according to the creation scenario of the brane universe. In this sense, we do not have to be afraid of this singularity, or equivalently, the singularity in Eq. (2), seriously. Moreover, when we consider the characteristic initial value problem, the initial conditions are chosen on a null hypersurface and the seam singularity is contained in its causal past. Therefore, the difficulties in the treatment of the singularity in 
Eq. (2) are simply reduced to the choice of initial conditions for gravitational waves as seen in below.

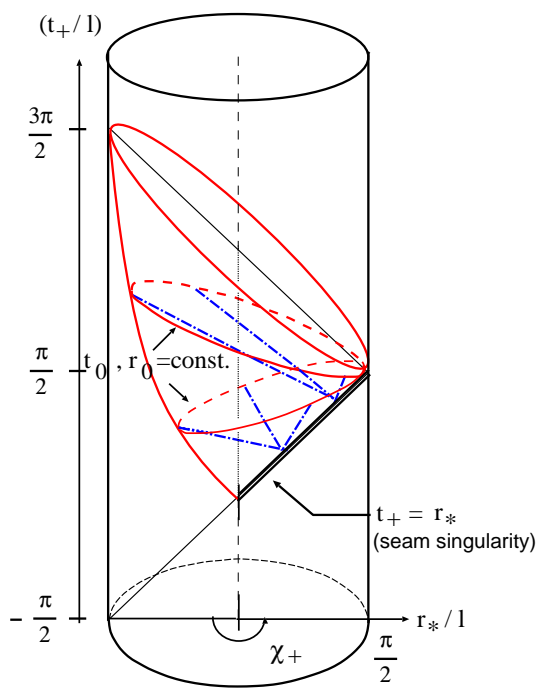

FIG. 2: Geodesics normal to the brane for each $\tau$ (dot-dashed lines) in the entire $\mathrm{AdS}_{5}$ spacetime. The geodesics converge to the seam singularity $t_{+}=r_{*}$ at which $\varphi(\tau, w)=0$.

\section{NULL HYPERSURFACE AND CAUSALITY IN THE BULK}

To clarify the causal structure of the brane universe and the domain of dependence of gravitational waves, let us now consider the future directed radial null geodesics from the brane into the bulk. These null geodesics determine the boundary associated with the causal region of the events on the brane.

In terms of the flat chart, the equations of these null geodesics are given by

$$
\frac{d t_{0}}{d \lambda}=\frac{l^{2}}{r_{0}^{2}} E, \quad \frac{d r_{0}}{d \lambda}=-E, \quad \frac{d \chi_{0}}{d \lambda}=0,
$$

where $E(>0)$ is an integration constant and $\lambda$ is the affine parameter along the geodesics. Because we concentrate on the radial null geodesics from the brane, $\chi_{0}$ is constant along the geodesics and the remaining equations in Eqs. (21) yield

$$
t_{0}=\frac{l^{2} E \lambda}{r_{b}\left(-E \lambda+r_{b}\right)}+t_{b}, \quad r_{0}=-E \lambda+r_{b} .
$$

In Eqs. (22), $t_{b}$ and $r_{b}$ are constants which correspond to the initial condition for the null geodesics. We regard that the point $\left(t_{b}, r_{b}\right)$ is on the brane in the flat chart and this point is the starting point of the geodesics. For this reason, we regard $t_{b}$ and $r_{b}$ as functions of the proper time $\tau$ on the brane and these functions are constrained by the generalized Friedmann equation Eq. (5).
Here, we note that the flat chart of $\mathrm{AdS}_{5}$ has the future Cauchy horizon in the entire $\mathrm{AdS}_{5}$. This Cauchy horizon appears as the "infinity" $\left(t_{0}, r_{0}\right) \rightarrow(+\infty,+0)$ in the flat chart. The above radial null geodesics from the brane approaches to this Cauchy horizon as the affine parameter $\lambda$ increases. We choose the affine parameter $\lambda$ so that $\lambda=0$ corresponds to the point on the brane and $\lambda=\frac{E}{r_{b}}>0$ corresponds to that on the Cauchy horizon.

On the other hand, in the closed chart, these null geodesics are given by

$$
\begin{aligned}
& r_{+}^{2}=\frac{1}{4}\left[\left(1+\frac{\zeta^{2}}{l^{2}}-\chi_{0}^{2}\right) r_{0}+2 \zeta\right]^{2}+r_{0}^{2} \chi_{0}^{2} \\
& \tan \left(\frac{t_{+}}{l}-\pi\right)=\frac{l}{l^{2}+\zeta r_{0}}\left\{\zeta-\frac{1}{2}\left(1-\frac{\zeta^{2}}{l^{2}}+\chi_{0}^{2}\right) r_{0}\right\} \\
& \cos \chi_{+}=\frac{1}{2 r_{+}}\left[\left(1+\frac{\zeta^{2}}{l^{2}}-\chi_{0}^{2}\right) r_{0}+2 \zeta\right]
\end{aligned}
$$

where $\zeta:=-\frac{l^{2}}{r_{b}}+t_{b}$. In Eqs. (23), $t_{0}$ and $r_{0}$ are parameterized by $\lambda$ through Eqs. (22). In the limit where the null geodesics from the brane approach to the Cauchy horizon $\left(t_{0}, r_{0}\right) \rightarrow(+\infty,+0)$, Eqs. (23) behave

$$
r_{+}=|\zeta|, \quad \frac{t_{+}}{l}=\arctan \left[\frac{\zeta}{l}\right]+\pi, \quad \cos \chi_{+}=\operatorname{sgn}(\zeta) .
$$

These do represent a point on the future Cauchy horizon of the flat chart.

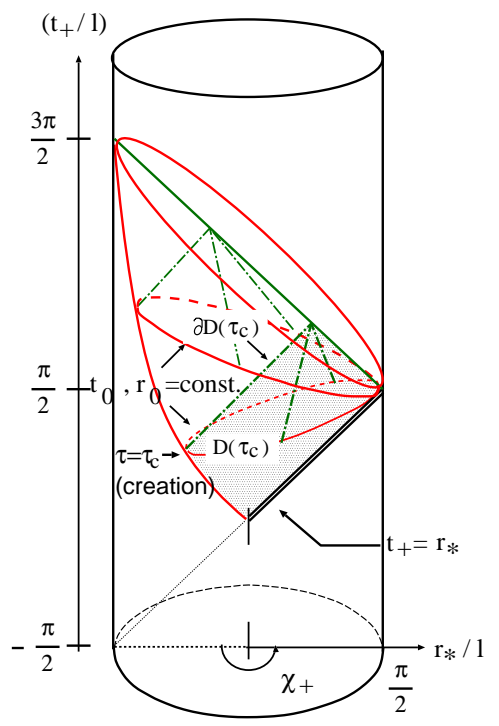

FIG. 3: Brane trajectory (lines) and future null geodesics from the brane (dot-dashed lines). Singularity where $\varphi\left(\tau, w_{h}\right)=0$ is shown as a thick line, which is entirely contained in the shaded region $\mathcal{D}\left(\tau_{c}\right)$. The evolution of gravitational waves is determined by boundary conditions on the brane and an initial spectrum on $\partial \mathcal{D}\left(\tau_{c}\right)$. The initial spectrum is determined by the creation scenario of the brane universe.

Actually, from the closed chart metric (3) with $K=$ +1 , we can easily see that the event represented by 
Eqs. (24) is pointlike when we fix the proper time $\tau$ on the brane. We also note the fact that Eqs. (24) do not depend on $\chi_{0}$. This implies that all null geodesics with different $\chi_{0}$ from the same $\tau=$ const. hypersurface on the brane reach to the same point represented by Eqs. (24). These null geodesics generate a null hypersurface. Hence we have one-to-one correspondence between a $\tau=$ const . hypersurface on the brane and a null hypersurface in the bulk. We denote this null hypersurface associated with $\tau=$ const. hypersurface on the brane by $\partial \mathcal{D}(\tau)$. We also denote the causal past of $\partial \mathcal{D}(\tau)$ by $\mathcal{D}(\tau)$. We note that $\mathcal{D}\left(\tau^{\prime}\right) \subset \mathcal{D}(\tau)$ for any $\tau^{\prime}<\tau$. In particular, the bulk region covered by the flat chart is foliated by the set of null hypersurfaces $\{\partial \mathcal{D}(\tau) \mid \tau$ is the proper time on the brane $\}$. Since $\partial \mathcal{D}\left(\tau^{\prime}\right) \subset \mathcal{D}(\tau)$ for $\tau^{\prime}<\tau$, the seam singularity, which is also represented by $\partial \mathcal{D}(\tau \rightarrow+0)$, is entirely contained in $\mathcal{D}(\tau)$ for any $\tau$. These situations are schematically depicted in Fig. 3.

Inspecting this causal structure, we can formulate the characteristic initial value problem of gravitational waves. Suppose that the flat FRW brane universe is created at the instance $\tau=\tau_{c}$. As depicted in Fig. 3] the initial state of gravitational waves in $\mathcal{D}\left(\tau_{c}\right)$ affects to their evolution only through the null hypersurface $\partial \mathcal{D}\left(\tau_{c}\right)$. Once we specify the state of gravitational waves in the region $\mathcal{D}\left(\tau_{c}\right)$ according to the creation scenario of the flat FRW brane, we can specify the spectrum of gravitational waves on $\partial \mathcal{D}\left(\tau_{c}\right)$. Hence, to determine the evolution of the spectrum of gravitational waves after $\tau=\tau_{c}$, we only have to specify the spectrum of gravitational waves on $\partial \mathcal{D}\left(\tau_{c}\right)$ as an initial condition and trace the evolution of them through Eq. (2) as seen in the next section.

\section{EVOLUTION OF GRAVITATIONAL WAVES}

At this point, we have a clear strategy to tackle the problem of the evolution of gravitational waves in the brane universe. The singularities $\varphi\left(\tau, w_{h}\right)=0$ in Eq. (2) are entirely contained in $\mathcal{D}(\tau)$, and the evolution of gravitational waves are determined by an initial spectrum on its boundary $\partial \mathcal{D}(\tau)$ and boundary conditions on the brane. Then, we do not have to care the singularity $\varphi\left(\tau, w_{h}\right)=0$ any more and there is no need to introduce any artificial boundaries in the bulk to impose boundary conditions at bulk infinity. In the following subsections, we first develope the formulation to obtain the numerical solutions to Eq. (2) in the context of brane world cosmology (in Sec. IVA), and then, we show numerical examples which are obtained by applying the formulation developed here (Sec. IVB).

\section{A. Numerical Formulation}

In order to develop the characteristic initial value problem associated with the null hypersurface $\partial \mathcal{D}(\tau)$, we in- troduce a null coordinate and rewrite down the equation of gravitational waves in a single null coordinate system

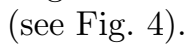

Now, we introduce the function $u$ by

$$
u=t_{0}-\frac{l^{2}}{r_{0}}-t_{b}^{\prime}+\frac{l^{2}}{r_{b}^{\prime}}
$$

where $\left(t_{b}^{\prime}, r_{b}^{\prime}\right)$ determine the zero point of this function $u$. The function $u$ is constant on the null hypersurface $\partial \mathcal{D}(\tau)$ for each $\tau$. We can easily confirm that the oneform $(d u)_{a}$ is null through the metric (3) with $K=0$.

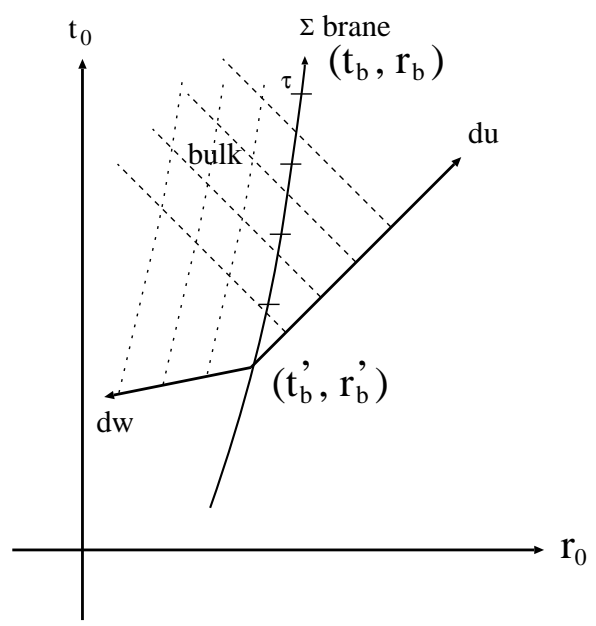

FIG. 4: Null coordinate and Gaussian normal coordinate in the flat chart.

Using the relations listed in the appendix (Eq. A6)(A9), see also Ref. 11]), the derivative $d u$ is given by

$$
\begin{aligned}
d u & =\left(\frac{\partial u}{\partial \tau}\right)_{w} d \tau+\left(\frac{\partial u}{\partial w}\right)_{\tau} d w \\
& =\frac{1}{\dot{a}}\left(\frac{\partial r_{0}}{\partial \tau}\right)_{w} F^{-1}(\tau, w) d \tau-F^{-1}(\tau, w) d w
\end{aligned}
$$

where

$$
F(\tau, w)=\frac{f\left[r_{0}(\tau, w)\right]}{\sqrt{f\left[r_{0}(\tau, w)\right]+\dot{a}^{2}}+\dot{a}} .
$$

In terms of the new coordinate system $u$ and $\bar{w}=w$, the five-dimensional metric (11) on $\mathrm{AdS}_{5}$ is written as

$$
d s^{2}=-F(\tau, \bar{w}) d u^{2}-2 F(\tau, \bar{w}) d u d \bar{w}+r_{0}^{2}(\tau, \bar{w}) d \Sigma_{0}^{2},(28)
$$

As mentioned in Sec. [1 the equation for gravitational waves in the bulk is simply given by that for the fivedimensional massless scalar field Eq. (2). In terms of the coordinate system $(u, \bar{w})$ Eq. (2) is given by 


$$
\left[\partial_{\bar{w}}^{2}-\frac{2}{F} \partial_{\bar{w}} \partial_{u}-\frac{3}{F r_{0}}\left(\left(\frac{\partial r_{0}}{\partial u}\right)_{\bar{w}} \partial_{\bar{w}}+\left(\frac{\partial r_{0}}{\partial \bar{w}}\right)_{u} \partial_{u}\right)+\left(\frac{1}{F}\left(\frac{\partial F}{\partial \bar{w}}\right)_{u}+\frac{3}{r_{0}}\left(\frac{\partial r_{0}}{\partial \bar{w}}\right)_{u}\right) \partial_{\bar{w}}-\frac{k^{2}}{r_{0}^{2}}\right] h(u, \bar{w} ; k)=0
$$

where $-k^{2}$ is the eigen value of the Laplacian of $d \Sigma_{0}$.

Eq. (29) can be formally integrated so that

$$
\begin{aligned}
\partial_{u} h(u, \bar{w}) & =r_{0}^{-3 / 2}(\tau, \bar{w}) \\
& \times\left(\int^{\bar{w}} S\left(w^{\prime}\right) r_{0}^{3 / 2} d w^{\prime}+C(u)\right),
\end{aligned}
$$

where

$$
\begin{aligned}
S(\bar{w}) & =\frac{F}{2}\left(\partial \bar{w} h-\frac{k^{2}}{r_{0}^{2}} h\right) \\
& +\left(\frac{1}{2} \frac{\partial F}{\partial \bar{w}}+\frac{3 F}{2} \frac{\partial \ln r_{0}}{\partial \bar{w}}-\frac{3}{2} \frac{\partial \ln r_{0}}{\partial u}\right) \partial_{\bar{w}} h
\end{aligned}
$$

The quantities $\partial r_{0} / \partial u, \partial r_{0} / \partial \bar{w}$ and $\partial F / \partial \bar{w}$ which appear in the definition (31) are given by known functions as

$$
\begin{aligned}
& \left(\frac{\partial r_{0}}{\partial u}\right)_{\bar{w}}=\dot{a} F \\
& \left(\frac{\partial r_{0}}{\partial \bar{w}}\right)_{u}=\dot{a}+\left(\frac{\partial r_{0}}{\partial w}\right)_{\tau} \\
& \left(\frac{\partial F}{\partial \bar{w}}\right)_{u}=\dot{a}\left(\frac{\partial r_{0}}{\partial \tau}\right)_{w}^{-1}\left(\frac{\partial F}{\partial \tau}\right)_{w}+\left(\frac{\partial F}{\partial w}\right)_{\tau}
\end{aligned}
$$

These equations (30) and (31) are the main result of this paper. In these equations, initial data of gravitational waves are set by choosing the function of $h(u, \bar{w})$ on $\partial \mathcal{D}(\tau)$ where the null coordinate $u$ is constant on $\partial \mathcal{D}(\tau)$. The function $C(u)$ in Eq. (30) is determined by the boundary condition at the brane $(\bar{w}=0)$ by

$$
\partial_{u} h(u, 0)=r_{0}^{-3 / 2}(\tau, 0) C(u) .
$$

When the anisotropic stress on the brane is not induced due to matter fields on the brane, the boundary condition for gravitational waves $h$ at the brane is the Neumann type [3]. This is accomplished by imposing

$$
\begin{aligned}
\partial_{u} h(u, 0) & =-\left(\frac{\partial u}{\partial w}\right)_{\tau}^{-1} \partial_{\bar{w}} h \\
& =F(\tau, 0) \partial_{\bar{w}} h,
\end{aligned}
$$

at the brane. Eq. (30) with the boundary condition (36) are easier to solve than Eq. (2) and enough to predict the cosmological evolution of gravitational waves. Note that our method have an important advantage that application to high energy epochs is easy and straightforward. Actually, this advantage is seen in the numerical examples shown in the following subsection (see Figs. [5] and 6).

\section{B. Numerical Scheme and Examples}

Here, we show some numerical solutions to Eq. (2) in the context of the brane world cosmology. We first comment on some details of the numerical scheme to obtain solutions to Eq. (29) with the boundary condition Eq. (36).

To solve Eq. (29), we evaluate Eq. (30) on each null hypersurface $\partial \mathcal{D}(\tau(u, \bar{w}=0))$, numerically. Once $h(u, \bar{w})$ on $\partial \mathcal{D}(\tau(u, \bar{w}=0))$ is given as a function of $\bar{w}$, we can evaluate $h(u+d u, \bar{w})$ on $\partial \mathcal{D}(\tau(u+d u, \bar{w}=0))$ by

$$
h(u+d u, \bar{w})=h(u, \bar{w})+d u \partial_{u} h(u, \bar{w}) .
$$

Through this evaluation on each $\partial \mathcal{D}(\tau(u, \bar{w}=0))$, we obtain the gravitational waves $h(u, \bar{w})$ in the causal future of the initial surface $\partial \mathcal{D}\left(\tau_{i n i}\right)$ with an appropriate initial condition. By evaluating $h(u, 0)$ on the brane, we see the behavior of gravitational waves on our brane universe.

To obtain $\partial_{u} h(u, \bar{w})$ by Eq. (30), we have to evaluate Eqs. (32)-(34) on each $\partial \mathcal{D}(\tau(u, \bar{w}=0))$ and Eq. (36) at the brane. To accomplish this, we evaluate $\dot{a}(\tau)=$ $a(\tau) H(a(\tau)), F(\tau, \bar{w})$, and $r_{0}(\tau, \bar{w})$ on each $\partial \mathcal{D}(\tau(u, \bar{w}=$ $0)$ ). Since these functions depend on $u$ only through $\tau$, we evaluate the change of the function $\tau(u, \bar{w})$ on each $\partial \mathcal{D}(\tau(u, \bar{w}=0))$. Further, it is also convenient to use the scale factor $a(\tau)$ at the brane as an time coordinate when we clarify the behavior of gravitational waves during the cosmic expansion.

We evaluate the change of the function $a(\tau(u, \bar{w}))$ on $\partial \mathcal{D}(\tau(u, \bar{w}=0))$ from Eq. (26) by setting $d u=0$, which yields

$$
\left(\frac{d \ln a}{d w}\right)_{u}=a H^{2}\left(\frac{\partial r_{0}}{\partial \tau}\right)_{w}^{-1}
$$

where $\left(\partial r_{0} / \partial \tau\right)_{w}$ is directly given by Eq. (9). By integrating Eq. (38) with the boundary condition $a(\tau)=$ $r_{0}(\tau, w=0)$ at the brane, we obtain the function $a(\tau(u, \bar{w}))$ along $\partial \mathcal{D}(\tau(u, \bar{w}=0))$. Since the relation between $a$ and $\tau$ is given by the explicit integration of the generalized Friedmann equation Eq. (5), we can evaluate $F(\tau, w)$ and $r_{0}(\tau, w)$ on each $\partial \mathcal{D}(\tau(u, \bar{w}=0))$ by using Eqs. (5) and (9). The relation between the time step $d u$ and $d \tau$ on the brane is given by

$$
d \tau=\dot{a}\left(\frac{\partial r_{0}}{\partial \tau}\right)_{w}^{-1} F(\tau, w=0) d u,
$$

which is led from Eq. (26) by choosing $d w=0$ and $w=0$. After proceeding the time step, we repeat the above evaluation of $a(\tau)$ and $\tau(u, \bar{w})$ on $\partial \mathcal{D}(\tau(u+d u, \bar{w}=0)$. Thus, 
we can evaluate $F(\tau, \bar{w}), r_{0}(\tau, \bar{w})$, and $\dot{a}$ on each null hypersurface $\partial \mathcal{D}(\tau(u, \bar{w}=0))$ from Eqs. (5), (9), and (26), and hence, Eqs. (32)-(34). Through these evaluations, we obtain $\partial_{u} h(u, \bar{w})$ by Eq. (30) and gravitational wave $h(u, \bar{w})$ in the causal future of the initial surface $\partial \mathcal{D}\left(\tau_{i n i}\right)$.

When we carry out this numerical scheme, we have to specify the zero point of the function $u$. Suppose that we start our numerical calculation from the high energy epoch $\left(l^{2} H^{2} \gg 1\right.$, or the $\rho^{2}$ dominated era). In this epoch, the generalized Friedmann equation and Eq. A3 lead

$$
\begin{aligned}
H^{2} & \sim \frac{1}{16} \tau^{-2} \\
\frac{a}{a_{0}} & =\left(\frac{2}{3} \kappa_{5}^{2} \rho_{\gamma 0} \tau\right)^{1 / 4}:=\alpha \tau^{1 / 4}, \\
t_{b}(\tau)-t_{b, \text { ini }}^{\prime} & \sim-\frac{l^{2}}{\alpha a_{0}}\left(\tau^{-1 / 4}-\tau_{\text {ini }}^{-1 / 4}\right),
\end{aligned}
$$

where $\rho_{\gamma 0}$ and $a_{0}$ are the energy density of radiations and the cosmic scale factor on the brane at the present universe. $t_{b, \text { ini }}^{\prime}$ is the trivial initial value of $t_{b}$ and we choose so that $t_{b, \text { ini }}^{\prime}=0$. Since the zero points of $u$ and $t_{b}$ are arbitrary, we choose so that $u=t_{b}^{\prime}=0$ and $r_{b}^{\prime}=$ $a\left(\tau_{\text {ini }}\right)$ in Eq.25) at the starting point of our numerical calculation $\tau=\tau_{\text {ini }}=1 / 4 H\left(a_{\text {ini }}\right)$.

Following to these numerical scheme, we obtain numerical examples depicted in Figs. 5] and [6] In these examples, we simply choose $h=$ const. as initial conditions of $h$ on the initial null surface $\partial \mathcal{D}\left(\tau_{\text {ini }}\right)$. We take this initial condition only as an example to demonstrate the calculation and investigate how the evolution of gravitational waves can be modified in high energy epochs qualitatively. The wave number is chosen $k=a\left(\tau_{\text {ini }}\right) H\left(\tau_{\text {ini }}\right)$, which corresponds to the mode just crossing the cosmic horizon at $\tau=\tau_{\text {ini. }}$. In Figs. [5] and [6] the scale factors of our examples are taken to be $a\left(\tau_{\text {ini }}\right)=1.58 \times 10^{-17}$ and $a\left(\tau_{\text {ini }}\right)=1.58 \times 10^{-16}$, respectively. The expansion of the universe is completely dominated by radiation in such early epochs. We only consider ordinary radiation (CMB) and three species of massless neutrinos for simplicity neglecting other matter components, and set the curvature scale of AdS5 bulk to be $1 \mathrm{~mm}$. In this cosmology, the transition from the $\rho^{2}$ dominated universe to the standard radiation dominated one occurs at $a \approx 4 \times 10^{-17}$.

The evolution of gravitational waves in brane world differs that of standard cosmology mainly in two points. First, the amplitude of gravitational waves in brane world models becomes smaller than that in four dimensional standard models. This is due to the fact that decelerating brane motion excites the modes propagating into the bulk 5]. Second, the effective frequency $\omega$ of gravitational waves measured on the brane becomes larger due to their momentum not only along the three dimensional space $(k$ in Eq.(29) ) but also to the bulk direction. These effects becomes remarkable in the high energy epoch $\left(l^{2} H^{2} \gg\right.$
1).

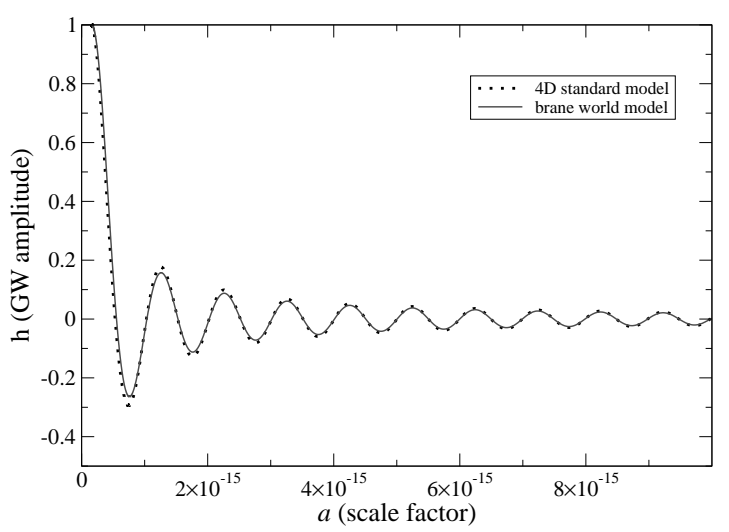

FIG. 5: Evolution of gravitational waves on the brane in brane world cosmology (solid line) which cross the horizon in low energy epochs $\left(l^{2} H^{2} \ll 1\right)$. Also shown is standard $4 \mathrm{D}$ evolution of gravitational waves (dotted line).

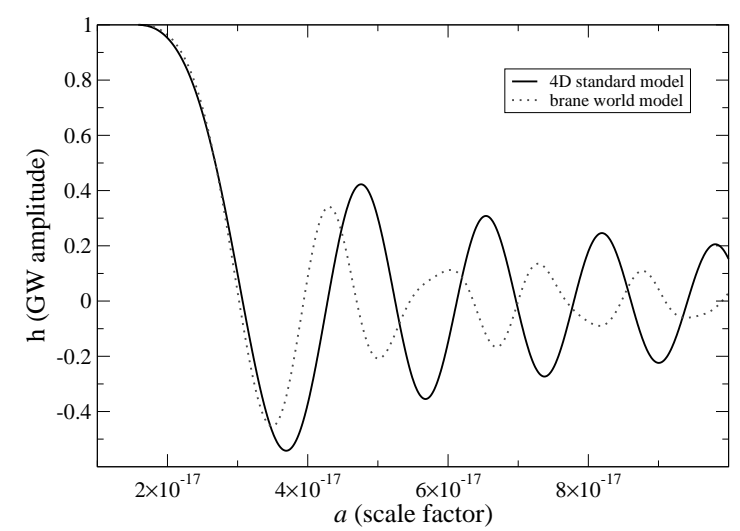

FIG. 6: The same as Fig. [5 but for gravitational waves which cross the horizon in high energy epochs $\left(l^{2} H^{2} \gg 1\right)$.

\section{SUMMARY AND DISCUSSION}

In this paper, we have carefully investigated the causal structure of the flat FRW model of RS type II brane world and proposed the single null coordinate system to solve the cosmological evolution of gravitational waves. We have explicitly seen that in this null coordinate system, we do not have to care the singularity in Eq. (2). Further, it is not necessary to introduce any artificial boundaries to impose some boundary conditions at the bulk infinity. Thus, we have shown that the problems of the singularity in Eq. (2) and the boundary conditions for gravitational waves at the bulk infinity are resolved if we simply choose an appropriate initial conditions for gravitational waves.

The initial condition for gravitational waves which we considered to obtain the numerical examples (Fig[5] and 
6) in the main text might not be realistic one, since $h=$ const. is not an exact solution to Eq.(29) but a mere approximated solution in the long wavelength limit $k \rightarrow 0$. Since the aim of this paper is to propose a numerical procedure to solve the evolution of cosmological gravitational waves, the details and quantitative studies of numerical results and the problem on realistic initial conditions of stochastic gravitational waves are beyond the current scope of this paper and they will be investigated in our forthcoming paper [12]. However, it is interesting to discuss the evolution of gravitational waves with an appropriate initial conditions and clarify the final spectrum of stochastic gravitational waves resulting from various creation scenarios of the brane world.

The initial conditions for gravitational waves in brane world cosmologies crucially depend on the creation scenario of the FRW brane. Many kinds of cosmological scenarios have been proposed so far [13]. Among them, there are some scenarios where the FRW brane is created after the inflationary phase. If we adopt these brane inflationary scenarios, it might be natural to consider that the initial spectrum of gravitational waves is determined in this inflationary phase. The spectrum of gravitational waves under the deSitter evolution of the brane is discussed by several authors [14, 15, 16]. It was pointed out that gravitational waves decay away except but the zero-mode, and it approaches to a constant amplitude in the inflationary phase [14]. These discussions are based on the GN coordinate system. Since GN coordinate system does not cover the entire bulk space, these discussions seem inappropriate to specify the initial spectrum of gravitational waves. However, it was also shown that the vacuum defined on the deSitter slicing asymptotically approaches to the vacuum defined in terms of the Poincare coordinates on $\mathrm{AdS}_{5}$ 15. This will imply that the vacuum state on the static $\mathrm{AdS}_{5}$ frame is appropriate as the bulk initial spectrum of gravitational waves when we consider these inflationary scenarios.

If we do not adopt the inflationary scenarios, we have to specify the initial spectrum according to the other creation scenario of the FRW brane. However, in any case, once given the initial configuration on a null hypersurface, our method can be applied to solve the evolution of gravitational waves. The final spectrum of the stochastic gravitational waves can be a powerful probe to investigate the existence of extra-dimensions by comparison with the spectrum in the four-dimensional standard cosmology. We leave this to future works.

Besides the evolution of stochastic gravitational waves, the procedure developed here will be applicable to discuss the evolution of the density perturbations in the brane world. The problem in the choice of the initial spectrum of the density perturbation will also arise as discussed above and this initial spectrum will also depend on the creation scenario of the FRW brane. However, according to the causality discussed in this paper, we can easily expect that the density perturbation is completely determined by the initial condition on a null hypersurface and the boundary conditions at the brane. Though this expectation should be confirmed by examining the equations for the density perturbations of the brane world, it is quite interesting to compare the evolution of the density perturbations in the brane world scenario with that in the conventional four-dimensional cosmology. We also leave this problem as a future work.

\section{Acknowledgments}

We would like to thank H. Ishihara and T. Tanaka for useful suggestions. This work has been supported in part by the Sasakawa Scientific Research Grant from The Japan Science Society. We also thank anonymous referees for pointing out our misleading presentations and improving the quality of our paper. KN would like to thank all members of Department of Physics of Hiyoshi Campus in Keio University, all members of Division of Theoretical Astronomy in NAOJ, and the other colleague for their continuous encouragement.

\section{APPENDIX A: GAUSSIAN NORMAL GEODESICS}

We briefly review the relation between the Gaussian normal (GN) coordinates and the flat chart in $\mathrm{AdS}_{5}$. To do this, we first consider the spacelike geodesics normal to the brane. Let us consider the unit normal vector $n^{a}\left(g_{a b} n^{a} n^{b}=1\right)$ tangent to these spacelike geodesics. The existence of the Killing field $\left(\partial / \partial t_{0}\right)^{a}$ in the bulk spacetime ensures that the existence of the constant $\mathcal{E}=$ $-g_{a b} n^{a}\left(\partial / \partial t_{0}\right)^{b}$ along the geodesics. Using this constant, the components of the normal vector $n^{a}$ are given by

$$
\begin{aligned}
n^{a} & =: n^{t_{0}}\left(\frac{\partial}{\partial t_{0}}\right)^{a}+n^{r_{0}}\left(\frac{\partial}{\partial r_{0}}\right)^{a} \\
& =\frac{\mathcal{E}}{f\left(r_{0}\right)}\left(\frac{\partial}{\partial t_{0}}\right)^{a}-\sqrt{f\left(r_{0}\right)+\mathcal{E}^{2}}\left(\frac{\partial}{\partial r_{0}}\right)^{a} .
\end{aligned}
$$

The explicit orbit of these geodesics is given by the integration of $d x^{\mu}=n^{\mu} d w$. For $r_{0}$ component, we obtain

$$
2 r_{0}^{2}+l^{2} \mathcal{E}^{2}=l^{2} \mathcal{E}^{2} \cosh \left[-\frac{2}{l}\left(w-w_{0}\right)\right]
$$

where $w_{0}$ is the additional constant of integration. We choose $\mathcal{E}$ and $w_{0}$ by the conditions: (i) the geodesics are normal to the brane world volume of $r_{0}=R\left(t_{0}\right)=a(\tau)$ at $t_{0}=t_{b}$ (i.e. $n_{a} \propto \nabla_{a}\left[r_{0}-R\left(t_{0}\right)\right]$ ); (ii) $w=0$ on the hypersurface. Since $d t_{0} / d \tau$ along the brane is given by the definition of the cosmic time $\tau$ :

$$
\begin{aligned}
-d \tau^{2} & =-f\left(r_{0}\right) d t_{0}^{2}+f^{-1}\left(r_{0}\right) d r_{0}^{2} \\
& =-f\left(r_{0}\right) d t_{0}^{2}+f^{-1}\left(r_{0}\right) \dot{a}^{2} d \tau^{2},
\end{aligned}
$$


the above two conditions lead

$$
\begin{aligned}
\mathcal{E}\left(t_{b}\right) & =-\dot{a}(\tau), \\
w_{0}\left(t_{b}\right) & =\frac{l}{2} \cosh ^{-1}\left[\frac{2 a^{2}+l^{2} \mathcal{E}^{2}\left(t_{b}\right)}{l^{2} \mathcal{E}^{2}\left(t_{b}\right)}\right] .
\end{aligned}
$$

Thus, substituting eqs. A4 into eq. A2 , we obtain eq. (9). On the other hand, the integration of the $t_{0}$ component of $d x^{\mu}=n^{\mu} d w$, i.e.,

$$
\frac{d t_{0}}{d w}=n^{t_{0}}=-\frac{l^{2} \dot{a}(\tau)}{\varphi(\tau, w) a^{2}(\tau)} .
$$

leads Eq. (10).

Finally, we summarize some useful relations. The fact that $\tau$ is constant along these spacelike geodesics leads

$$
\begin{aligned}
& \left(\frac{\partial t_{0}}{\partial w}\right)_{\tau}=n^{t_{0}}=-\frac{\dot{a}}{f\left(r_{0}\right)}, \\
& \left(\frac{\partial r_{0}}{\partial w}\right)_{\tau}=n^{r_{0}}=-\sqrt{f\left(r_{0}\right)+\dot{a}^{2}} .
\end{aligned}
$$

Also, from the definition that $d w=g_{\mu \nu} n^{\mu} d x^{\nu}$, we obtain

$$
\begin{aligned}
& \left(\frac{\partial w}{\partial t_{0}}\right)_{r_{0}}=\dot{a}(\tau), \\
& \left(\frac{\partial w}{\partial r_{0}}\right)_{t_{0}}=-\frac{\sqrt{f\left(r_{0}\right)+\dot{a}^{2}(\tau)}}{f\left(r_{0}\right)} .
\end{aligned}
$$

These relations are used in the main text (see SecIV).
[1] L. Randall and R. Sundrum, Phys. Rev. Lett. 83, 4690 (1999) arXiv:hep-th/9906064.

[2] P. Binetruy, C. Deffayet, U. Ellwanger and D. Langlois, Phys. Lett. B 477, 285 (2000) arXiv:hep-th/9910219; P. Kraus, JHEP 9912, 011 (1999) arXiv:hep-th/9910149; S. Mukohyama, Phys. Lett. B 473, 241 (2000) arXiv:hep-th/9911165.

[3] H. Kodama, A. Ishibashi and O. Seto, Phys. Rev. D 62, $064022(2000)$

[4] D. Langlois, R. Maartens, M. Sasaki and D. Wands, Phys. Rev. D 63, 084009 (2001) arXiv:hep-th/0012044; K. Koyama and J. Soda, Phys. Rev. D 65, 023514 (2002) arXiv:hep-th/0108003.

[5] T. Hiramatsu, K. Koyama and A. Taruya, Phys. Lett. B 578, 269 (2004) arXiv:hep-th/0308072.

[6] R. Easther, D. Langlois, R. Maartens and D. Wands, JCAP 0310, 014 (2003) arXiv:hep-th/0308078.

[7] H. Ishihara, Phys. Rev. D 66, 023513 (2002) arXiv:gr-qc/0107085 ; H. Ishihara and I. Tanaka, Proceedings of the 5th RESCEU, p59 (2001) .

[8] K. Nakamura, Phys. Rev. D 66, 084005 (2002) arXiv:gr-qc/0205031.

[9] K. Ichiki, M. Yahiro, T. Kajino, M. Orito and
G. J. Mathews, Phys. Rev. D 66, 043521 (2002) arXiv:astro-ph/0203272.

[10] D. N. Spergel et al., to be appear in ApJ, arXiv:astro-ph/0302209

[11] S. Mukohyama, T. Shiromizu and K. i. Maeda, Phys. Rev. D 62, 024028 (2000) [Erratum-ibid. D 63, 029901 (2001)] arXiv:hep-th/9912287.

[12] K. Ichiki and K. Nakamura, arXiv:astro-ph/0406606].

[13] J. Khoury, B. A. Ovrut, P. J. Steinhardt and N. Turok, Phys. Rev. D 64, 123522 (2001) arXiv:hep-th/0103239; J. J. Blanco-Pillado and M. Bucher, Phys. Rev. D 65, 083517 (2002) arXiv:hep-th/0111089; J. Garriga and T. Tanaka, Phys. Rev. D 65, 103506 (2002) arXiv:hep-th/0112028; U. Gen, A. Ishibashi and T. Tanaka, Phys. Rev. D 66, 023519 (2002) arXiv:hep-th/0110286 .

[14] D. Langlois, R. Maartens and D. Wands, Phys. Lett. B 489, 259 (2000) arXiv:hep-th/0006007.

[15] D. S. Gorbunov, V. A. Rubakov and S. M. Sibiryakov, JHEP 0110, 015 (2001) arXiv:hep-th/0108017.

[16] T. Kobayashi, H. Kudoh and T. Tanaka, Phys. Rev. D 68, 044025 (2003) arXiv:gr-qc/0305006. 